\title{
The Glass Transition of Aliphatic Ionenes*
}

\author{
Adi Eisenberg, Hiroshi Matsuura, ${ }^{* *}$ and Tetsuo Yokoyama*** \\ McGill University, Montreal, Canada. \\ (Received May 15, 1970)
}

\begin{abstract}
The glass-transition temperatures of a wide range of ionenes have been studied as a function of the spacing of ions and the dielectric constant of the plasticizer. For solvents of a high dielectric constant the extrapolated glass transition of most ionenes has a value of $c a .0^{\circ} \mathrm{C}$, while for plasticizers of a low dielectric constant the value centers around $-80^{\circ} \mathrm{C}$. It is suggested that a conformational transition is responsible for this drastic change in the extrapolated $T_{g}$ values. A $q / a$ effect was also found, the slope of the $T_{g} v s . q / a$ plot being 695 , i.e., quite similar to that for the phosphates, silicates, and acrylates.
\end{abstract}

KEY WORDS Glass Transition / Ionic Polymers / Aliphatic Ionenes / Spacing of Ions / Dielectrics / Plasticizer / Conformation /

While it is known that the incorporation of ionic species into polymers influences their properties profoundly, ${ }^{1,2}$ the only systematic study of the effect of ions on the glass-transition temperature of polymers has been performed on an inorganic system, the polymetaphosphates. ${ }^{2}$ It is true that several studies have been performed on organic systems containing ions at relatively low concentration, ${ }^{4-8}$ but the incorporation of a high concentration of ions into organic polymers yields highly intractable materials with decomposition temperatures frequently lying below $T_{g} .{ }^{9}$ The polyphosphates represent an inorganic system in which, as a result of the occurrence of bond interchange at high temperatures, ${ }^{10}$ no decomposition occurs even in completely ionic systems, and for this reason that system was selected for the first extensive study.

The aliphatic ionenes, ${ }^{11}$ the structure of which is given in Figure 1, represent an organic polymer in which the spacing of the ions is uniform and can be varied widely. These

* Different aspects of this work were presented, in part, at two ACS symposia dealing with ionic polymers and were partly preprinted in Polymer Preprints, 9, 617 (1968) and 10, 861 (1969).

** On leave from the Nippon Oil Seal Industry, Co., Ltd., Japan.

*** Present Address: Department of Applied Chemistry, Kyushu University, Japan.

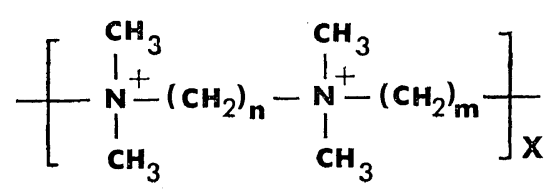

Figure 1. $n, m$ ionene.

materials, therefore, represent an excellent system in which the effect of ions on the glass-transition temperature of organic polymers can be explored extensively. Specifically, one can inquire into the following factors:

(1) Variation of $T_{g}$ with average spacing of ions along the chain. Both $n$ and $m$ can be varied, and thus the average spacing can be easily changed.

(2) Effect of the uniformity of the spacing of ions along the chain on $T_{g}$. An average of 6 methylene units between ions can be achieved, for instance, by making both $m$ and $n$ equal to 6 or by making $m=4$ and $n=8$.

(3) The plasticizing effect of solvents of a range of dielectric constants on the glass transition of the plasticized polymer. Solvents have to be used because the ionenes are highly crystalline and the only way to cbtain a $T_{g}$ value for the pure material is to measure $T_{g}$ for samples plasticized to various degrees and and to extrapolate to zero plasticizer content. Since the ionenes possess both strongly hydrophilic and hydrophobic segments, it is reason- 
able to expect that various plasticizers will influence the polymer differently.

(4) The effect of the structure of the nonionic part of the backbone. It is possible to prepare branched ionenes by using appropriate materials, and the effect of this structural variation is also of interest.

(5) Finally, it would be useful to inquire to what extent the generalizations that have been found from previous studies of ionic polymers (mostly salts of copolymers of acids, or homopolymeric acids) are applicable to materials possessing large quaternary ammonium cations along the backbone and large, highly polarizable counterions (bromide ions were used exclusively in this study).

Since ref 8 provides a brief but very recent review of the effect of ionic forces on the glass transition of polymers, the subject matter will not be dwelt on here. It should suffice to say that for low concentrations of ions in organic polymers, ${ }^{4 \sim 7}$ the glass transition increases practically linearly with concentration; the linear increase seems to be independent of the mode of incorporation of the ions, i.e., they can be present either in the form of a comonomer or as a dissolved salt (for instance lithium perchlorate in polypropylene glycol). The effectiveness of the salt in raising the glass-transition ranges from $c a$. 3.1 to 9.7 degrees per mol $\%$ of the ionic component.

In the studies of the polyphosphate ${ }^{3}$ and silicate $^{12}$ systems, it was found that the glass- transition temperature is linearly related to the ratio of the cation charge $(q)$ to the internuclear distance between cation and anion at closest approach $(a)$. For polyphosphosphates, the equation is

$$
T_{g}=625 q / a-12
$$

while for the silicates it is

$$
T_{g}=635 q / a+132
$$

where $q$ is expressed in units of one electron and $a$ in $\AA$. A similar relation also seems to be valid for the acrylates, ${ }^{13}$ i.e.,

$$
T_{g}=730 q / a-67
$$

It is of interest therefore to see whether the ionenes also yield a similar relation.

\section{EXPERIMENTAL}

\section{Procedures}

Materials. The materials used in the polymerization were $\alpha-\omega$ dibromoalkanes and tetramethyldiamino alkanes. In most cases, unbranched methylene sequences were utilized; for some studies, however, branched dibromides or diamines were used. The materials were obtained from Aldrich Chemical Company and, wherever possible, purified by distillation under reduced pressure. Dibromodecane, -dode cane, and -hexadecane were purified by triple recrystallization from hot ethanol, with sub-

\begin{tabular}{|c|c|c|c|c|c|c|c|c|c|c|}
\hline & 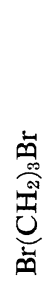 & 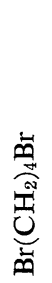 & 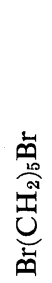 & 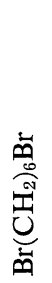 & 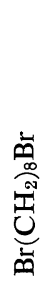 & 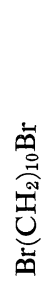 & 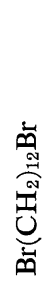 & 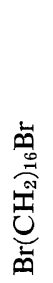 & 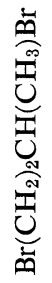 & 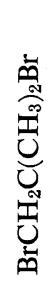 \\
\hline$\left(\mathrm{CH}_{3}\right)_{2} \mathrm{~N}\left(\mathrm{CH}_{2}\right)_{6} \mathrm{~N}\left(\mathrm{CH}_{3}\right)_{2}$ & $\bigcirc$ & $\bigcirc$ & $\bigcirc$ & $\bigcirc$ & & $\bigcirc$ & 0 & 0 & & \\
\hline$\left(\mathrm{CH}_{3}\right)_{2} \mathrm{~N}\left(\mathrm{CH}_{2}\right)_{4} \mathrm{~N}\left(\mathrm{CH}_{3}\right)_{2}$ & $\bigcirc$ & 0 & $\bigcirc$ & & $\bigcirc$ & $\bigcirc$ & $\bigcirc$ & & & \\
\hline$\left(\mathrm{CH}_{3}\right)_{2} \mathrm{~N}\left(\mathrm{CH}_{2}\right)_{2} \mathrm{CH}\left(\mathrm{CH}_{3}\right) \mathrm{N}\left(\mathrm{CH}_{3}\right)_{2}$ & $\bigcirc$ & 0 & 0 & $\bigcirc$ & $\bigcirc$ & & & & $\bigcirc$ & O \\
\hline
\end{tabular}
sequent drying under vacuum.

Table I. ${ }^{\text {a }}$ Tabulation of all ionenes used in the study

a Polymers containing less than 3 methylene sequences in any of the starting materials were not studied, since Rembaum, et al., ${ }^{14}$ showed that materials of very low $n$ or $m$ values did not yield polymers. 
Dimethylformide(DMF) and methanol or water were used as solvents, and both were distilled prior to use. Glycerine (Allied Chemical) and ethylene glycol (Matheson Coleman \& Bell) were used without further purification.

Table I presents in tabular form all the materials which were prepared and studied in the course of this work.

Polymerization. The polymerizations were carried out in DMF-methanol-(50:50 vol \%) mixtures, except for the $6,6,6,8$, and 6,10 ionenes which were polymerized in DMF-water mixtures containing $10 \sim 30 \%$ water by volume. No drastic difference between the polymers obtained from these two solvent systems were observed, except that the average intrinsic viscosities of those polymerized in DMF-water tended to be somewhat higher than those of the other group. All the polymerizations were carried out at room temperature, the polymerization time being around 30 days. Intrinsic viscosities of the 6 series centered on $0.15 \mathrm{dl} / \mathrm{gm}$. (in $0.4-M \mathrm{KBr}$ ) while those of the 4 series centered on 0.05 in the same solvent.

Sample Preparation. The water plasticized samples were prepared by adding excess water (to dissolve the ionene) and evaporating till a predetermined weight was reached. The evaporations were carried out under atmospheric pressure between 25 and $40^{\circ} \mathrm{C}$. The samples plasticized with glycerine were prepared in exactly the same way, except that evaporations were carried out at $50^{\circ} \mathrm{C}$. under partial vacuum. Finally, the samples plasticized with glycerine-water mixtures were prepared by dissolving a known amount of the ionene in a 20-\% glycerine-water solution and evaporating at $25^{\circ} \mathrm{C}$. The assumption was made that no glycerine was lost during the course of the evaporation. While this is not absolutely correct, the ratio of water to glycerine over mixtures of the two is so high that only a negligible amount of the glycerine could have evaporated.

$T_{g}$ Determinations. All the glass transitions were determined on a Perkin-Elmer DSC-I instrument, at scanning speeds of $10^{\circ} \mathrm{C} / \mathrm{min}$.

\section{Experimental Results}

Water Plasticized materials. The plot of $T_{g}$ $v s$. wt $\%$ water for the 6,8 ionene is shown in

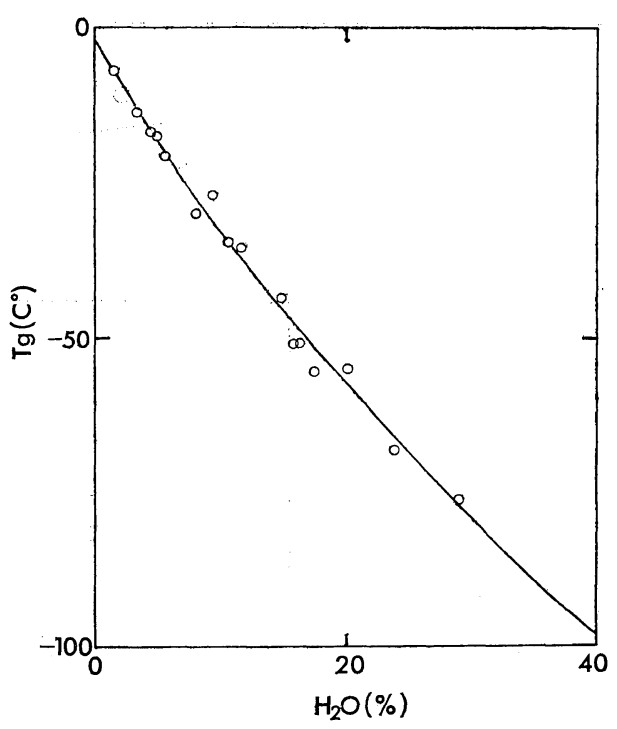

Figure 2. Glass transition temperature for water plasticized 6,8 ionene: $\bigcirc$, experimental; - calculated from $T_{g}=269 W_{1}+127 W_{2}-158 W_{1} W_{2}$.

Figure 2. The 6,8 ionene was chosen for presentation in this figure because it was the most thoroughly studied polymer and the largest number of pcints were obtained for it. The points are experimental, and the line is calculated from the relation ${ }^{15}$

$$
T_{g}=w_{1} T_{g_{1}}+w_{2} T_{g_{2}}+K w_{1} w_{2}
$$

where $w$ represents the weight fraction, $K$ a constant and the subscripts 1 and 2 refer to polymer and solvent respectively. The glasstransition temperature for pure water was taken as $127^{\circ} \mathrm{K}$, a value obtained by J. Yannas ${ }^{16}$ by extrapolation of the $T_{g}$ values of the waterglycerine system, to zero glycerine content, and subsequently confirmed in our own work. Since $T_{g}, w_{1}, w_{2}$, and $T_{g_{2}}$ are known, $T_{g_{1}}$ and $K$ can be obtained from the equation by plotting $\left(T_{g}-T_{g_{2}}\right) / w_{1} v s . w_{2}$, from the intercept and slope, respectively. For the 6, 8 system, $T_{g_{1}}=269^{\circ} \mathrm{K}$ and $\mathrm{K}=-158 \mathrm{deg}$. The results for all the systems are presented graphically in Figure 3, where the glass transition is plotted against the average spacing of the ions along the backbone.

It is noteworthy that the glass transition of the 6,4 ionenes is very much lower than would be expected by comparison with materials of 


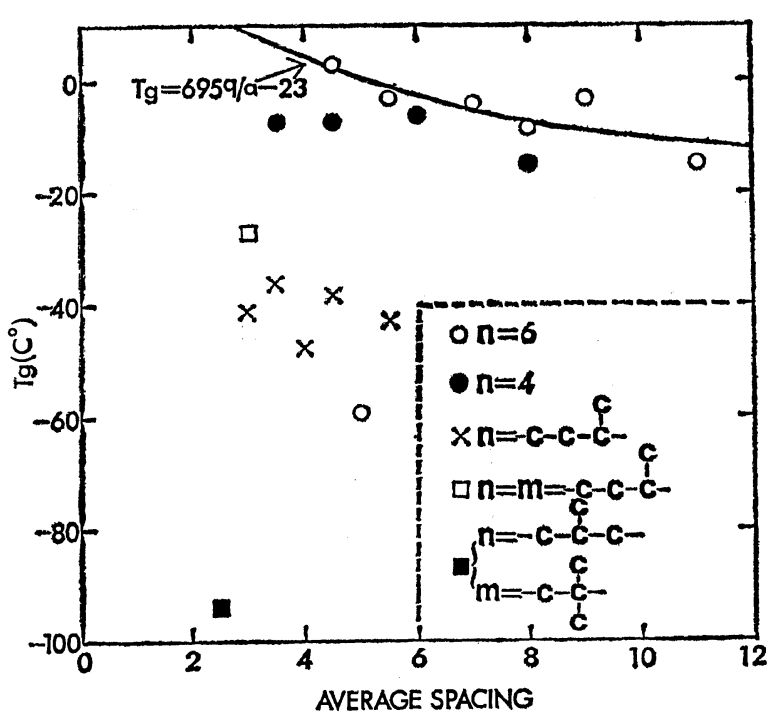

Figure 3. Glass-transition temperatures of all ionene samples (determined with water as plasticizer and extrapolated to zero water content). The average spacing was calculated from $(n+m) / 2$.

similar composition, it is $c a .-59^{\circ} \mathrm{C}$. The $T_{g}$ of the 4,4 or 6,6 ionenes could not be determined at all.

The branched ionenes seem to have a much lower glass-transition temperature; the results are also shown in Figure 3.

Glycerine Plasticized Materials. The table below shows the glass transition as a function of composition for several glycerine plasticized ionenes. The 6,4 or 6,6 ionenes seem to exhibit no abnormality here. However, all the extrapolated $T_{g}$ values are $c a .80^{\circ}$ lower than those obtained with water as a plasticizer.

\begin{tabular}{cc}
\hline Material & $T_{g}$ \\
\hline $6-3$ & $-80^{\circ} \mathrm{C}$ \\
$6-4$ & $-84^{\circ} \mathrm{C}$ \\
$6-5$ & $-79^{\circ} \mathrm{C}$ \\
$6-6$ & $-81^{\circ} \mathrm{C}$ \\
$6-8$ & $-82^{\circ} \mathrm{C}$ \\
$6-10$ & $-79^{\circ} \mathrm{C}$ \\
\hline
\end{tabular}

Other Pure Plasticizers. In addition to water and glycerine, formamide, ethylene glycol, and methanol were also used as plasticizers for the 6,8 ionene system. The results were

$\begin{array}{ll}\text { Formamide } & -18^{\circ} \mathrm{C} \\ \text { Methanol } & -75^{\circ} \mathrm{C} \\ \text { Ethylene Glycol } & -91^{\circ} \mathrm{C}\end{array}$

Glycerine-Water Mixitures as Plasticizers. Since it was evident that different plasticizers gave remarkably different extrapolated $T_{g}$ values for the same material, for instance $-4 v s .-83$ for the 6,8 ionone with water or glycerine, it was of interest to determine the effect of mixed plasticizers, for instance water-glycerine mixtures. The results are shown in Figure 4, indicating a steady decrease in $T_{g}$ with increasing glycerine content.

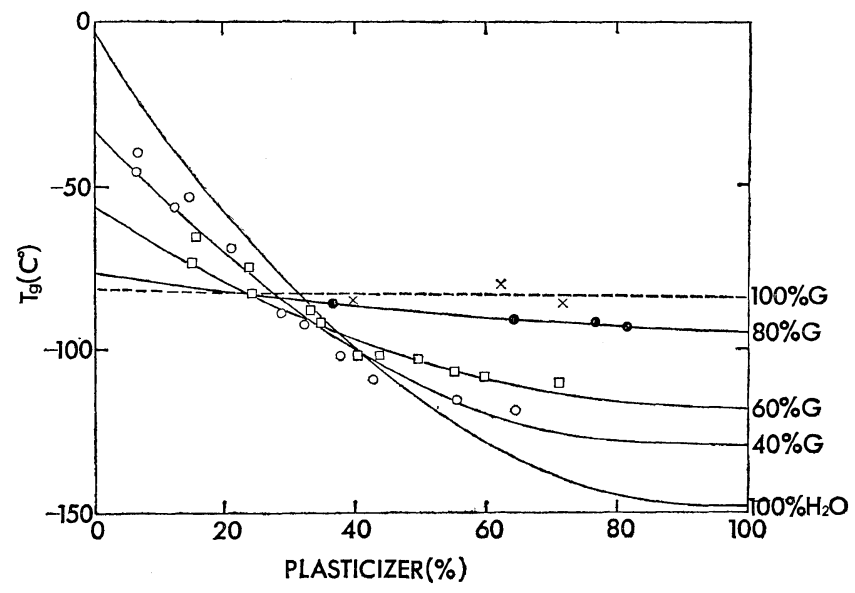

Figure 4. Glass-transition temperatures for 6, 8 ionene plasticized with waterglycerine mixtures: $\bigcirc$, experimental; - calculated using data. The composition of the plasticizer is given on the right. 
The extrapolated values of the glass transition and $K$ values are

\begin{tabular}{lcccc}
\hline Solvent composition & $\begin{array}{c}\text { Extra- } \\
\text { polated } \\
T_{g} \\
\text { (0\% solvent) }\end{array}$ & \multicolumn{2}{c}{$\begin{array}{c}T_{g}(0 \% \\
\text { solvent) } \\
-T_{g} \\
\text { (solvent) }\end{array}$} \\
\hline 100\% glycerine & -82 & -1.3 & 2 \\
$80 \%$ glycerine-20\% water & -76 & -15 & 18 \\
$60 \%$ glycerine-40\% water & -57 & -61 & 61 \\
$40 \%$ glycerine-60\% water & -31 & -122 & 99 \\
$0 \%$ glycerine-100\% water & -4 & -158 & 142 \\
\hline
\end{tabular}

The table also lists the difference in the glasstransition temperatures between the polymer and solvent. This difference will be discussed later.

The extrapolated values of the glass transitions of 6,8 ionene with all the plasticizers which were studied are plotted in Figure 5 against

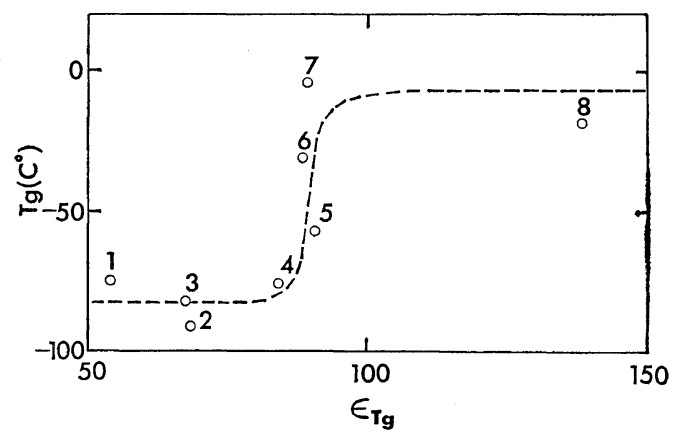

Figure 5. Glass transition of 6,8 ionene (extrapolated to zero plasticizer content) using various plasticizers, plotted against the dielectric constant of the plasticizer at $T_{g}$ : 1, methanol; 2, ethylene glycol; 3, glycerine; 4, 80\% glycerine-20\% water; 5 , 60\% glycerine-40\% water; $6,40 \%$ glycerine$60 \%$ water; 7 , water; 8 , formamide.

the dielectric constant of the pure plasticizer at the extrapolated $T_{g}$ value. The values of the dielectric constant were taken either from the Handbook of Chemistry and Physics or from the papers of Akerlof, ${ }^{17}$ plotted as a function of temperature, and extrapolated to $T_{g}$.

\section{DISCUSSION AND CONCLUSIONS}

\section{The Effect of Solvents}

The most significant result of this study is undoubtedly the finding of the large discrepancy in extrapolated $T_{g}$ values of the 6,8 ionene, depending on the dielectric constant of the plasticizer. The most reasonable explanation of this phenomenon is based on the assumption of a conformational change of the ionene caused by a change in the dielectric constant of the liquid which is used as a plasticizer. Most probably, the conformation of the polymer in a relatively dilute water solution and in the highly concentrated noncrystalline form (either pure if it could be achieved that way or in the presence of a small amount of plasticizer) is similar and dominated both by the strongly hydrophobic and by the ionic segments of the chain. By contrast, in the presence of glycerine or other plasticizers of a low dielectric constant, the chain conformation could change; possibly, the chain expands because as a result of the presence of both hydrophobic and hydrophilic segments in glycerine (and the other alcohols), the hydroxyl groups can interact with the ions, thus converting the polymer into a hydrophobic entity.

It should be pointed out that explanations of the phenomenon which are based on specific site-bonding models, i.e., those which involve very specific interactions between the polymer and the diluent, are not sufficient to explain the phenomenon, although these interactions may be present. If the interactions were dominant then then plasticized polymer could be treated as a copolymer system, and with a decreasing diluent concentration one would have to get back to the $T_{g}$ of "pure" ionene, whatever it might be. The fact that a range of $T_{g}$ values is obtained must indicate that the polymer itself has changed, and a conformational change is the simplest possibility.

Several experimental facts seem to be able to be interpreted in the light of the above explanation. It should be pointed out that they neither support nor contradict the hypothesis of a conformational change, but merely that they can be explained, albeit speculatively, within that framework.

Branches on the main chain lower the glass transition appreciably. Since branching represents a barrier to internal rotation, the $T_{g}$ lowering seems to imply that that polymer is frozen into a low $\varepsilon$ conformation even in the presence 


\section{A. Eisenberg, H. Matsuura, and T. Yokoyama}

of water, otherwise $T_{g}$ would be expected to increase with the introduction of methyl groups along the main chain.

The glass transitions of the 4,4 and 6,6 ionenes with water cannot be determined because these materials crystallize readily; a DSC run reveals melting or freezing over very wide concentration ranges. However the $T_{g}$ of the 6,4 ionene can be determined, and yields a value of $c a$. $-59^{\circ} \mathrm{C}$ using water as a plasticizer. Apparently the high regularity of the chain again forces the polymer into a low $\varepsilon$ conformation.

\section{The q/a Effect}

It is of interest to inquire whether a $q / a$ effect exists in ionenes. First one has to calculate the $q / a$ value for each of the materials, and then plot the observed $T_{g}$ value against this $q / a$. To avoid errors due to possible slight differences in material characteristics (molecular weight, etc.) only one series of polymers was chosen for this study, the 6-ionenes, because more samples of that series were studied than of any other.

To determine the $q / a$ effect, we need to know first the size of the ions involved. The internuclear distance between bromine and nitrogen in crystalline tetramethylammonium bromide is $5 \AA^{17}$ which would make $q / a$ for the equivalent completely ionic polymer 0.2 if the $\mathrm{N}-\mathrm{Br}$ internuclear distance in the polymer and the crystal is the same. At this point, the assumption is made that, at least conceptually, every repeat unit of the chain can consist of $-\mathrm{N}\left(\mathrm{CH}_{3}\right)_{2}^{+}-$; this means that the $q / a$ value for a $n, m$ ionene would be $0.2 \times 2 /(n+m+2)$ since there are $n+m+2$ backbone atoms per repeat unit, two of which are ionic. Using the above approach and the $T_{g}$ values for the 6 series one obtains (by least squares) a line given by

$$
T_{g}=695 q / a-23
$$

If one were to assume, arbitrarily, that only every second atom along the backbone is ionizable, then the slope would decrease by a factor of two. It is, however, very significant that using the simplest assumption, one gets a slope which is close to that obtained for the phosphates, silicates, and acrylates, implying that for the ionene (at least in its high $\varepsilon$ conforma- tion), a $q / a$ effect does exist which in its magnitude is similar to that in the other organics and inorganics. It should be remembered, however, that the assumption that every ion is theoretically at least, ionizable, is arbitrary, so the absolute magnitude of the slope of $T_{g} v s$. $q / a$ is not as important as the fact that an approximately linear relation exists between $T_{g}$ and $q / a$. It is also significant that the extrapolated $T_{g}$ value for the completely nonionic material, i.e., pure polyethylene, is $c a .-25^{\circ} \mathrm{C}$, again for this particular conformation.

Arguments along the above lines cannot be made for glycerine plasticized polymer, due to the scatter of the data.

The Equivalence of $K$ and $\left(T_{2}-T_{1}\right)$

Jenckel and Heusch ${ }^{15}$ pointed out that for many polymer-diluent systems the value of $K$ is very close to the glass-transition temperature difference between polymer and diluent. We have found this to be the case quite precisely for the 6,8 series (see the table) independent of solvent, but not for any of the other series studied. No explanation for the inapplicability of the above correlation, for instance in the 6ionene-water series, can be given at this time.

Acknowledgements. The assistance of Mr. F. Baxter in determinating the $T_{g}$ of the 6,8-ionene -water-glycerine system is gratefully acknowledged.

This work was supported, in part, by the Petroleum Research Fund, administered by the American Chemical Society, and in part by the Office of Naval Research of the United States.

\section{REFERENCES}

1. Symposium on Ionic Polymers, held in conjunction with San Francisco ACS Meeting, San Francisco, Calif., April 1968; Polym. Preprints, 9, No. 1, 1968.

2. Symposium on Cationic Polymers, held in conjunction with New York ACS Meeting, New York, N.Y., Sept. 1969; Polym. Preprints, 10, No. 2, 1969.

3. A. Eisenberg, H. Farb, and L. G. Cool, J. Polym. Sci., Part A-2, 4, 855 (1966).

4. J. Moacanin and E. F. Cuddihy, J. Polym. Sci, Part C, 14, 313 (1966).

5. W. E. Fitzgerald and L. E. Nielsen, Proc. Roy. 
Soc., A 282, 137 (1964).

6. E. P. Otocka and F. R. Eirich, J. Poiym. Sci., Part A-2, 6, 921 (1968).

7. E. P. Otocka and T.K. Kwei, Macromolecules, 1, 401 (1968).

8. A. Eisenberg, "Glass Transitions in Ionic Polymers" Paper presented at the Joint Meeting of the ACS and CIC, Toronto, May 1970; Macromolecules, 4, 125 (1971).

9. L. E. Nielson, ref. 1, p 596.

10. A. Eisenberg, Adv. Polym. Sci., 5, 59 (1967).

11. A. Rembaum, W. Baumgartner, and A. Eisenberg, Polym. Letters, 6, 159 (1968).

12. A. Eisenberg and K. Takahashi, J. Non Cryst. Solids, 3, 279 (1970).
13. A. Eisenberg, H. Matsuura, and T. Yokoyama, in press.

14. H. Noguchi and A. Rembaum, Polym. Letters, 7, 383 (1969); A. Rembaum, S. Singer, and H. Keyzer, Polym. Letters, 7, 395 (1969), also other papers, in press.

15. E. Jenckel and R. Heusch, Kolloid Z, 130, 89 (1953).

16. I. Yannas, Science, 160, 298 (1968).

17. G. Akerlof, J. Amer. Chem. Soc., 54, 4125 (1932).

18. R. Wyckoff, "Crystal Structures"' Vol. I, 2nd ed., American Chemical Society Monographs Series, Chemical Catalog Co., 1935, p 107. 